\title{
CRYOGENIC CONTROLS FOR FERMILAB'S SRF CAVITIES AND TEST FACILITY
}

\author{
B. Norris, R. Bossert, A. Klebaner, S. Lackey, A. Martinez, L. Pei, \\ W. Soyars, V. Sirotenko
}

Fermi National Accelerator Laboratory

Batavia, IL, 60510, USA

\begin{abstract}
A new superconducting radio frequency (SRF) cavities test facility is now operational at Fermilab's Meson Detector Building (MDB). The facility is supplied cryogens from the Cryogenic Test Facility (CTF) located in a separate building 500-m away. The design incorporates ambient temperature pumping for super-fluid helium production, as well as three $0.6-\mathrm{kW}$ at $4.5-\mathrm{K}$ refrigerators, five screw compressors, a helium purifier, helium and nitrogen inventory, cryogenic distribution system, and a variety of test cryostats.

To control and monitor the vastly distributed cryogenic system, a flexible scheme has been developed. Both commercial and experimental physics tools are used. APACS ${ }^{\mathrm{TM}}$, a process automation control system from Siemens-Moore, is at the heart of the design. APACS $+{ }^{\mathrm{TM}}$ allows engineers to configure an ever evolving test facility while maintaining control over the plant and distribution system. APACS $+{ }^{\mathrm{TM}}$ nodes at CTF and MDB are coupled by a fiber optic network. DirectLogic205 PLC's by KOYO ${ }^{\circledR}$ are used as the field level interface to most I/O.

The top layer of this system uses EPICS (Experimental Physics and Industrial Control System) as a SCADA/HMI. Utilities for graphical display, control loop setting, real time/historical plotting and alarming have been implemented by using the world-wide library of applications for EPICS.

OPC client/server technology is used to bridge across each different platform.

This paper presents this design and its successful implementation.
\end{abstract}

KEYWORDS: Test facilities, RF cavities, Controls 


\section{INTRODUCTION}

Testing of superconducting RF modules at Fermilab is beginning, utilizing the International Linear Collider Test Area (ILCTA). The cryogenic systems, with reliable and flexible controls, are an important component. This paper will focus on the now operational cryogenic system controls for the Meson Detector Building (MDB). The general cryogenic system [1] and current operating experience [2] is presented elsewhere. The current operational loads are the Capture Cavity II, a nine-cell SRF accelerating cavity, and the Horizontal Test Cryostat (HTC), a platform for testing future SRF cavity assemblies.

\section{PHILOSOPHY OF MESON CRYOGENIC CONTROLS}

Today's controls scheme at CTF and MDB is the result of an evolutionary process that occurred in three distinct steps - marrying PLC's with Siemens APACS+ (distributed control system), the networking of two separate controls environments in two buildings 500-meters apart, and the use of EPICS as an HMI/SCADA.

\section{Step 1 - Marrying PLC’s with APACS}

In the late 1990's, efforts focused on upgrading an existing 4.5-K refrigeration system to a facility that could support 2.0-K operations (now referred to as CTF). As cryogenic upgrade efforts were on-going, a strategy for implementing new cryogenic controls at an old facility had to be implemented. Monetary and manpower constraints meant virtually all existing field devices/process equipment would need to be re-used (originally based on 1980's Tevatron design). Added to this constraint was the fact that the wiring of the original refrigeration system was failing and not well known by those still working at Fermilab.

Our solution was to break down the refrigerator and compressor systems into small self-contained controls objects. Major cryogenic components such as expansion engines, screw compressors, or a purifier were instrumented with specialized, dedicated PLC's. For example, an expansion engine has its own PLC which interfaces to a motor controller. Complete monitoring and control of this expander is accomplished with this localized PLC. Not wanting to repeat the original setup of large bundles of wires from field devices to a central computer, a fiber optic Ethernet solution created a network from a central computer to each PLC. Today, there are eleven PLC's operating at the CTF, each having a dedicated multi-mode fiber.

APACS $+{ }^{\mathrm{TM}}$ Process Automation System was chosen as the centerpiece computer of

this control solution. From this commercial distributed controls package, cryogenic engineers have the ability to configure and control the physical process as needs change. Control loops, automation, and a central database all reside at this level. OPC (Open Process Control) technology, operating on a dedicated PC, is used as the communication bridge from the field PLC's to APACS ${ }^{\mathrm{TM}}$.

This arrangement has now been operating reliably for approximately 6-7 years.

\section{Step 2 - A Network Solution to a Distributed Cryogenic System}

The second phase of the controls evolution came about when a second building (MDB) located 500-meters from CTF was chosen as the end-user location for the cryogens provided by CTF. Operational considerations required us to consider allowing for a control 


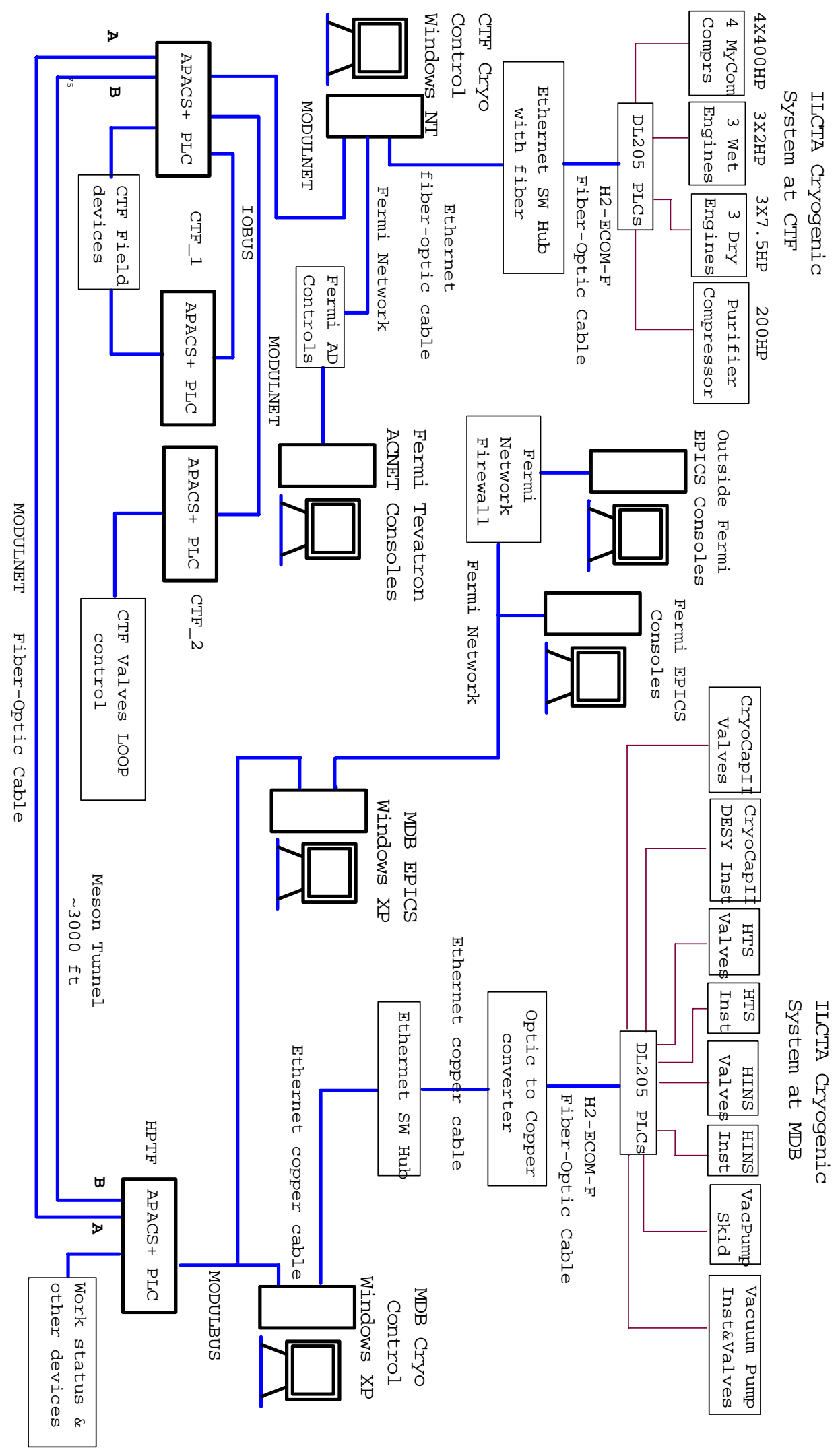

FIGURE 1. The ILCTA_MDB Cryogenic Control System. 
room in both the CTF and MDB, with configuration and control over the CTF plant and the MDB loads from either location. It quickly became apparent that a solution built on our CTF experiences was the correct solution.

A second APACS $+{ }^{\text {TM }}$ node was placed at MDB and a fiber network was established between the two central processors at each building (fiber routing approximately 1000-m). Siemen's Modulnet networking system uses redundant circuits, so two fiber circuits exist from CTF to MDB and provide the communication link from APACS $+{ }^{\mathrm{TM}}$ to APACS $+{ }^{\mathrm{TM}}$ nodes.

The MDB is a large end-use facility that, cryogenically speaking, has the ability to provide services for new cryomodule test caves as needs arise. We needed a control's solution that was as distributed as the cryogenic system itself. Again, an object view of cryogenic segments was chosen. Specialized PLC's were configured to support electric actuators while another PLC was designed for nominal cryogenic instrumentation. These PLC's can be replicated as the cryogenic usage expands. Further, the MDB contains an ambient temperature pump for super-fluid helium loads. A dedicated PLC supports field device readings, interlocks, and controls configuration.

In all cases, the PLC's were networked to the MDB APACS+ via individual multimode fiber optic links. There are now eight PLC’s operating at MDB.

\section{Step 3 - EPICS, the HMI/SCADA}

The final phase of creating this distributed controls scheme was to choose an HMI that would allow users to monitor and control the cryogenics for their experiment. Ease of use and familiarity was an important goal. Since this new cavity test facility is an international endeavor, high level lab management made a decision to choose controls which international users would be familiar. Many physics laboratories around the world use EPICS tools to create their control systems. The choice of EPICS allows people from other laboratories to bring EPICS-ready equipment they have developed at their facility and quickly and easily integrate into the controls system at the Fermilab superconducting RF test facility. For cryogenics, we decided to layer EPICS over APACS ${ }^{\mathrm{TM}}$ for our human interface package.

\section{CONTROLS SPECIFICS FOR CTF}

As mentioned previously, the CTF cryogenic system consists of three 0.6-kW refrigerators operating at 4.5-K. Each refrigerator consists of a heat exchanger, two expansion engines and a valve box. The expansion engines each have a dedicated PLC interfacing to the motor controller for complete monitoring and control of process alarms and trips associated with each expander. Each PLC is connected to the CTF APACS $+{ }^{\mathrm{TM}}$ controller via a fiber optic Ethernet connection. Field I/O such as pressure and temperature measurement as well as electric actuators are read and controlled directly using Siemens APACS $+{ }^{\mathrm{TM}} \mathrm{I} / \mathrm{O}$ modules.

The main CTF compressor system consists of four Mycom screw compressor skids, each driven by a 300-kW motor. A dedicated PLC for each compressor skid is the local control for all skid functions and also communicates with the main APACS ${ }^{\mathrm{TM}}$ node via a fiber optic Ethernet network. Field device readings such as pressure and temperature are read back directly as APACS $+{ }^{\mathrm{TM}}$ field I/O using Siemens APACS $+{ }^{\mathrm{TM}}$ voltage input and RTD modules.

Gaseous helium management is done using pneumatically controlled inventory control valves which regulate refrigerator suction and discharge by sending gas back and 
forth between the refrigerator and gas storage. The control valves are operated using I/P transducers which convert the 4-20 mA signals from the APACS $+{ }^{\mathrm{TM}}$ control system to 20.7 -103.4 kPa (3-15 psi) outputs to pneumatically control the valves.

With the addition of a helium vacuum pump for 1.8-K operation, a purifier compressor was also integrated into the system to boost the discharge pressure of the vacuum pump in order to send it through an in-line helium purifier. This was done in order to remove any potential impurities from the helium stream as a result of sub atmospheric operation prior to being reclaimed by the refrigeration system. The purifier compressor is a Mycom screw compressor skid driven by a $150-\mathrm{kW}$ motor. A dedicated PLC is used to control and monitor the compressor. Due to a limited number of available I/O channels from the APACS $+{ }^{\mathrm{TM}}$ control system, local PLC I/O channels were used for monitoring and control of temperature and pressure process points and communicated back to the APACS $+{ }^{\text {TM }}$ control system via the fiber optic connection.

\section{PLC SPECIFICS FOR MDB}

The PLC systems at MDB are each dedicated to a specific area or function, the intention being that this provides the most flexibility and freedom to modify or repair a system without impacting operation of another. All PLCs are Automation Direct DL205 series with D2-260 CPUs. Each PLC has an Ethernet Communications Module for communicating to the main control system via fiber optic.

\section{Cavity Test Cryostats (for Cryocap II and Horizontal Test Cryostat)}

The Capture Cavity II cave controls consist of two PLCs. The first PLC is dedicated to the operation of eight electrically operated analog control valves. The valve position is read back with an LVDT. The front of the PLC cabinet has meters for local read back of the valve positions and switches for local/remote control and for open/close functions in the manual mode. The valves are fully controllable locally in the event of a PLC failure. The second PLC is dedicated to all other instrumentation needs which mainly consist of analog inputs for pressures and temperatures and discrete inputs to read the status of limit switches and similar hardware. A relay rack for support equipment to interface to field devices such as level probes and temperature sensors is located next to the instrumentation PLC. Both PLCs and most of the relay rack equipment are powered by an uninterruptible power supply (UPS) dedicated to the local area controls.

The HTC cave controls are nearly an exact copy of the Capture Cavity II cave controls. There is an electric valve control PLC cabinet, an instrumentation PLC cabinet and support equipment relay rack outfitted with very similar hardware.

\section{Helium Vacuum Pump}

The helium vacuum skid is used for pumping in order to achieve 1.8-K operation. The skid consists of a ring type pump with a 150-kW motor and a booster pump equipped with a 75-kW motor and variable frequency drive (VFD). A PLC is the local control of all skid functions and as an interface for remote control. It communicates via Modbus protocol to the VFD used to operate the booster. The PLC functions as the first line of defense in the interlocking and controlled shutdown functions of the skid in the event of a problem. The skid also has a backup hardwired relay chain which only operates on selected parameters 
TABLE 1. I/O Details

\begin{tabular}{|c|c|c|c|c|c|c|c|c|}
\hline DEVICE & $\begin{array}{c}\text { CC } \\
\text { II }\end{array}$ & HTC & $\begin{array}{c}\text { CAVITY } \\
\text { GAS } \\
\text { MANAGE }\end{array}$ & $\begin{array}{c}\text { VAC } \\
\text { PUMP }\end{array}$ & $\begin{array}{c}\text { REFRIG } \\
\text { x 3 }\end{array}$ & $\begin{array}{c}\text { COMPR } \\
\text { x 4 }\end{array}$ & $\begin{array}{c}\text { INVENTORY/ } \\
\text { MISC. }\end{array}$ & $\begin{array}{c}\text { PURIFIER } \\
\text { SYSTEM }\end{array}$ \\
\hline $\begin{array}{c}\text { Positive } \\
\text { pressure } \\
\text { transmitter }\end{array}$ & 13 & 4 & 3 & 3 & 6 & 4 & 11 & 5 \\
\hline $\begin{array}{c}\text { Vacuum } \\
\text { pressure } \\
\text { transmitter }\end{array}$ & 5 & 5 & 1 & 2 & 0 & 0 & 0 & 0 \\
\hline $\begin{array}{c}\text { Temperature } \\
\text { sensors }\end{array}$ & 20 & 23 & 2 & 2 & 10 & 2 & 10 & 7 \\
\hline $\begin{array}{c}\text { Misc. } \\
\text { Analog } \\
\text { transmitters }\end{array}$ & 13 & 12 & 6 & 2 & 19 & 4 & 30 & 9 \\
\hline $\begin{array}{c}\text { Oxygen } \\
\text { Deficiency } \\
\text { Safety } \\
\text { Transmitters }\end{array}$ & 6 & 6 & 4 & 0 & 2 total & 1 total & $\begin{array}{c}\text { Included in } \\
\text { Refrigerator } \\
\text { Total }\end{array}$ & 2 \\
\hline $\begin{array}{c}\text { Analog } \\
\text { Outputs }\end{array}$ & 1 & 2 & 3 & 0 & 0 & 0 & 0 & 0 \\
\hline $\begin{array}{c}\text { Digital } \\
\text { Inputs }\end{array}$ & 26 & 46 & 15 & 32 & 7 & 1 & 16 & 4 \\
\hline $\begin{array}{c}\text { Digital } \\
\text { Outputs }\end{array}$ & 30 & 20 & 4 & 21 & 12 & 2 & 25 & 8 \\
\hline \begin{tabular}{c} 
TOTAL \\
\hline
\end{tabular} & 114 & 118 & 38 & 62 & 164 & 53 & 92 & 35 \\
\hline
\end{tabular}

which have been determined to be critical. Under normal circumstances the PLC is always in control of all skid functions, however, if the PLC fails and doesn't act to shut down the skid, the hardwired relay interlock will crash the skid before damage can occur.

A small PLC is located somewhat midway between the superconducting RF caves area and the helium vacuum pump area (approximately 150-m separation from pump to caves). It is dedicated to all instrumentation and valve control needs that are not specific to either cave or the vacuum pump. Its main function is interfacing to analog control valves and other instrumentation for controlling the helium vacuum manifold. It also interfaces to utility support equipment for monitoring instrument air and cooling water.

\section{APACS}

The parallel APACS controllers with two level distribution control system are the center of the cryogenic control system. The two level distribution control system utilizes the Siemens Programmable Logic Controller (PLC) as the upper level PID loop control and the Automation Direct PLC as the lower level direct control. The two APACS $+{ }^{\mathrm{TM}}$ which are almost $1000 \mathrm{~m}$ apart share their system information through ModulNET.

The PID loop control adjusts its output based on its input, set point, sample time and error as input. For example, a critical tuning requirement is too maintain super fluid bath stability within +/- 0.1 torr through throttle valve control. FIGURE 2 highlights the normal response to a pressure disturbance. 


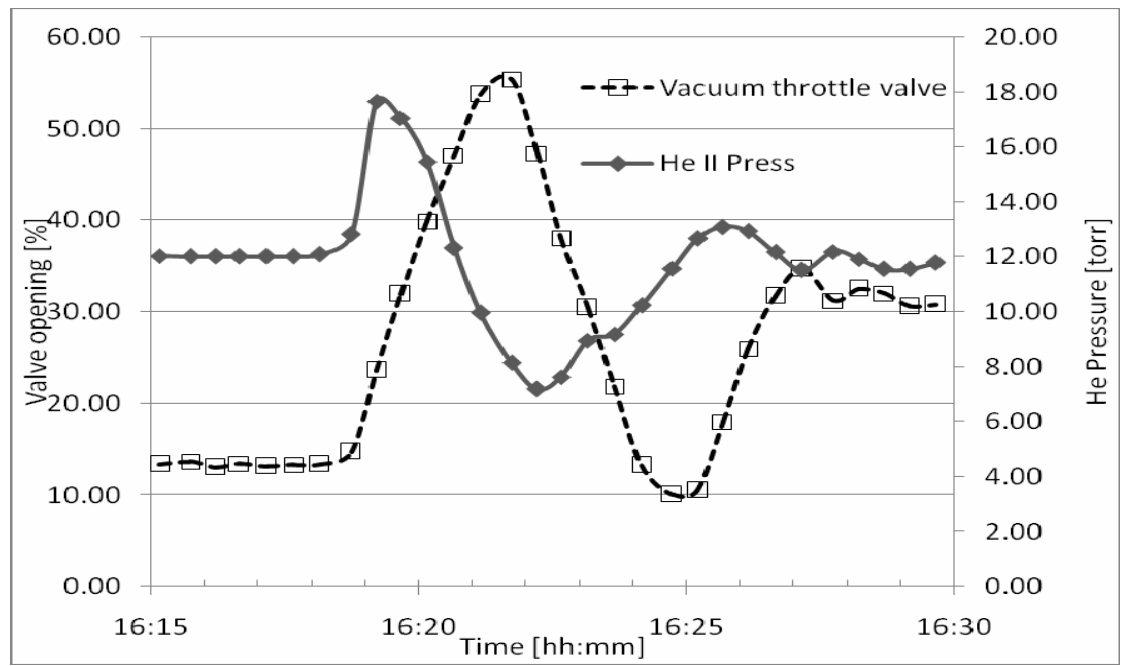

FIGURE 2. Capture Cavity II super fluid pressure control valve response to disturbance.

\section{EPICS}

The higher level control system user interfaces, with graphics capability and functionalities as alarms, archiving, plotting and save/restore current state features, are provided by the EPICS (Experimental Physics and Industrial Control System) tool kit ${ }^{3}$. Usage of EPICS allows us to bind all elements of the control system into the flexible distributed system with remote operator access. The EPICS tool kit was developed by a collaboration of physics laboratories and is available through the EPICS Open Source license. EPICS is separated into base functionality and extensions. EPICS base comprises

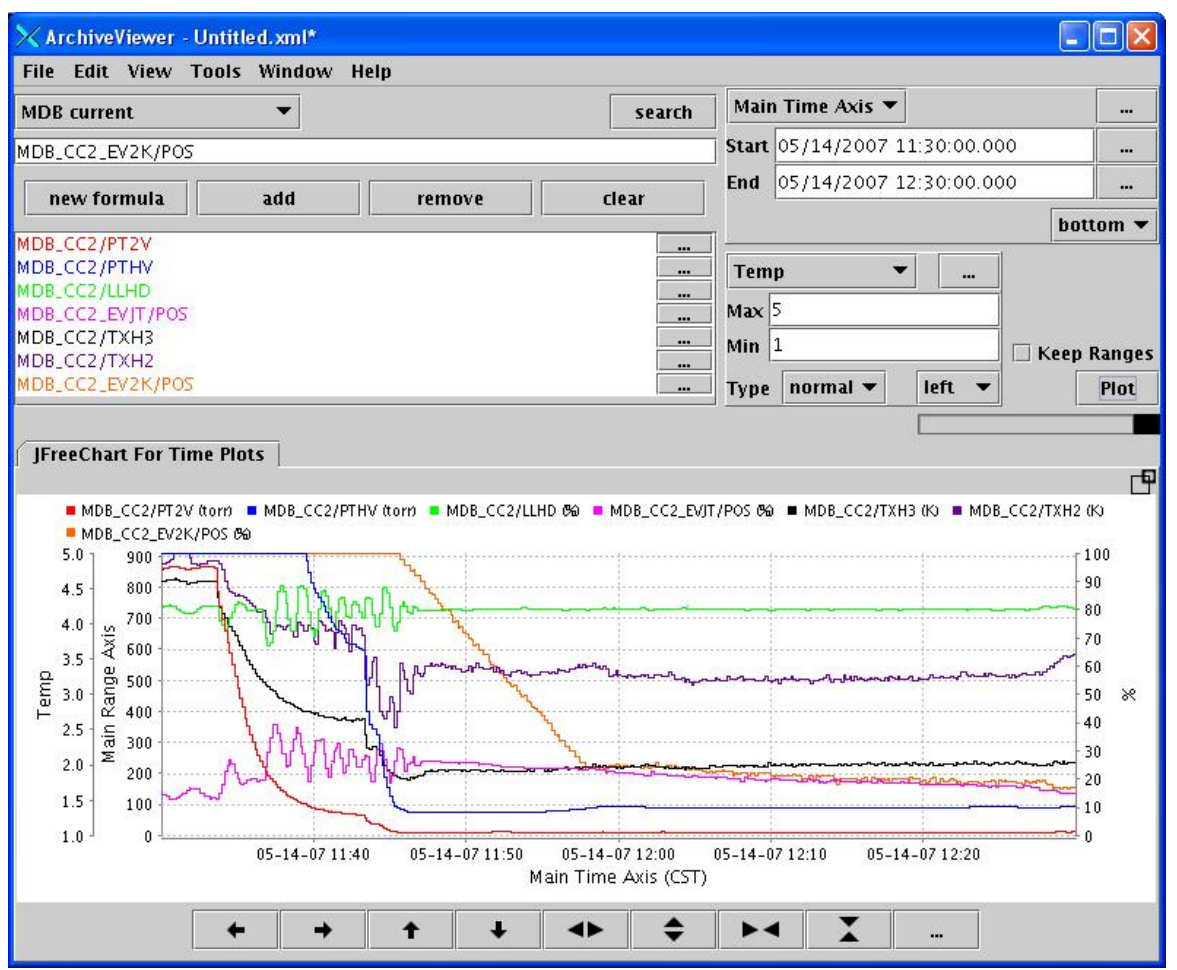

FIGURE 3. Archiver historical data plot from EPICS. 
core functionality including client/server communication via the EPICS channel access protocol on Ethernet, build system tools, database access and processing libraries and standard device types with support. EPICS extensions which are used in this system include alarms handling, data archiving, save and restore, graphical data display builders and plotting packages, and some proprietary scripts to support and maintain EPICS databases. For example, FIGURE 3 shows the EPICS extension Archiver which plots historical data, in this case a super fluid pump down of Capture Cavity II.

The EPICS control system accesses the APACS ${ }^{\text {TM }}$ OPC server (Open Process Control) via an EPICS software IOC (Input Output Controller) which runs as an OPC client on a Windows XP node ${ }^{4}$ and serves as a gateway between APACS $+{ }^{\mathrm{TM}}$ and EPICS worlds.

\section{CONCLUSIONS}

A new controls strategy has been implemented to support the needs of cryogenic engineers and end-users at Fermilab's SRF Cavities and Test Facility. Three levels of controls have been used - PLC's for field device interfacing, APACS $+{ }^{\mathrm{TM}}$ Process Automation System as a configuration tool, and EPICS as the HMI/SCADA for all real time data monitoring and control. A fiber optic hub allows communication and controls configuration to be done from two separate APACS $+{ }^{\mathrm{TM}}$ nodes which are in separate buildings 500-m apart.

The distributed strategy presented in this paper has been proven to be reliable and flexible allowing providing for growth and adaptation to new cryogenic equipment and end-users. At the time of this writing 19 PLC's, two APACS $+{ }^{\mathrm{TM}}$ nodes, and a dedicated EPICS IOC are successfully operating and providing users with a reliable cryogenic supply.

\section{ACKNOWLEDGEMENTS}

This work is supported by the U. S. Department of Energy under contract DE-AC0207CH11359. The authors express their thanks to all of the efforts from personnel from the $\mathrm{AD} /$ Cryogenic Department and the AD/Controls Department. It is the technicians of these groups that have successfully put this system together.

\section{REFERENCES}

1. Klebaner, A. L., and Theilacker, J. C., “Cryogenics for the Superconducting Module Test Facility,” in Advances in Cryogenics Engineering 51B, edited by J. G. Weisend, American Institute of Physics, Melville, New York, 2006, pp. 1428-1435.

2. Klebaner, A. L., et al., "Superconducting Radio-Frequency Modules Test Facility Operating Experience," submitted to Advances in Cryogenics Engineering 53, American Institute of Physics, Melville, New York. Submitted at this conference.

3. Experimental Physics and Industrial Control System, http://www.aps.anl.gov/epics.

4. Fleischhauer, et al., "Integrating PLCs with a OPC Interface into an EPICS Based Control System”, Proceedings of ICALEPCS2003, Gyeongju, Korea, pp 445-447. 